LAWRENCE LIVERMORE NATIONAL LABORATORY

\title{
Study of the Uncertainty on Compton Ring Reconstruction
}

J. Gronberg, S. Johnson, D. Lange, D. Wright

July 26, 2004 


\section{Disclaimer}

This document was prepared as an account of work sponsored by an agency of the United States Government. Neither the United States Government nor the University of California nor any of their employees, makes any warranty, express or implied, or assumes any legal liability or responsibility for the accuracy, completeness, or usefulness of any information, apparatus, product, or process disclosed, or represents that its use would not infringe privately owned rights. Reference herein to any specific commercial product, process, or service by trade name, trademark, manufacturer, or otherwise, does not necessarily constitute or imply its endorsement, recommendation, or favoring by the United States Government or the University of California. The views and opinions of authors expressed herein do not necessarily state or reflect those of the United States Government or the University of California, and shall not be used for advertising or product endorsement purposes.

\section{Auspices Statement}

This work was performed under the auspices of the U.S. Department of Energy by University of California, Lawrence Livermore National Laboratory under Contract W-7405-Eng-48. 
$\mathrm{h} 4 \mathrm{doc} 3 \mathrm{v} 2$

\title{
Study of the uncertainty on Compton ring reconstruction
}

\author{
Jeff Gronberg, Stephen Johnson, David Lange, Doug Wright \\ High Energy Physics group, N Division
}

September 24, 2003

\section{Introduction}

The purpose of this study was to estimate the effect of measurement errors in the position and energy of the observed interactions on the angular resolution of a Compton-ring imaging type detector.

In a Compton interaction, if one can measure the energy of the scattered photon and the energy of the Compton electron, then one can determine the scattering angle between the incoming and outgoing photon using the well-known Compton formula (see Appendix A.1 on page 6 for the derivation of this formula).

$$
\cos \theta=1+\frac{m_{e}}{E_{\gamma}}-\frac{m_{e}}{E_{\gamma^{\prime}}}
$$

where $E_{\gamma}=E_{\gamma^{\prime}}+E_{e}-m_{e}$.

If one assumes small Gaussian errors $\left(\delta E_{e}, \delta E_{\gamma}\right)$ for the observed energies $\left(E_{e}, E_{\gamma^{\prime}}\right)$, then one can derive an analytic expression for the error on the reconstruction angle $\theta$

$$
\delta \theta^{2}=\frac{m_{e}{ }^{2}}{\sin ^{2} \theta E_{\gamma}{ }^{4}}\left[\delta E_{e}{ }^{2}+\left(1-\frac{E_{\gamma}{ }^{2}}{E_{\gamma^{\prime}}{ }^{2}}\right)^{2} \delta E_{\gamma^{\prime}}{ }^{2}\right] .
$$

See Appendix A.2 (page 7) for the derivation of this result and a numerical cross-check. Note that this error function approaches infinity if $\theta$ is near zero or $\pi$. A detailed discussion on how to avoid this is given in Appendix A.2.1 (page 7) and the impact on the reconstruction of the simulated events is described in Section 4 (page 2).

\section{Error in the determination of the scattered photon direction}

There is an additional error on the determined Compton ring due to the uncertainty in the scattered photon direction. This is due to the finite precision on the two measured interaction points. In order to estimate this contribution to the Compton angle error, we use the following empirical procedure.

For each measured coordinate, we displace the nominal value by the expected measurement error in that coordinate and determine the change in the direction of the scattered photon, i.e., the opening angle between the nominal direction and direction determined with one coordinate shifted. We repeat this procedure for each of the six coordinates and add, in quadrature, the calculated angle shifts.

We have checked that this procedure agrees numerically with an analytic formulation of the error, see Equation 7 in Appendix B on page 9. The analytic formulation has the advantage that it is statistically meaningful. The input and output variables will have Gaussian distributions and thus one can calculate confidence limits. On the other hand, the results are Gaussian only when the fractional errors are less than about $50 \%$.

We combine, in quadrature, the angular error derived from the position measurement uncertainty (using the empirical method) with the error determined from Eq. 2 for the total $d \theta$ of the Compton ring.

\section{Simulation}

Using this information, we have determined how $d \theta$ depends on the detector resolution. We use a detector geometry consiting of a silicon slab that is $8 \mathrm{~cm}$ by $8 \mathrm{~cm}$ by $4 \mathrm{~cm}$. We use a point source of photons that is placed on an axi perpendicular to one of the $8 \mathrm{~cm}$ by $8 \mathrm{~cm}$ faces of this detector. We use Geant 4 to determine the event sample, considering only events where the full energy of the incoming photon has 


\section{Energy Resolution}

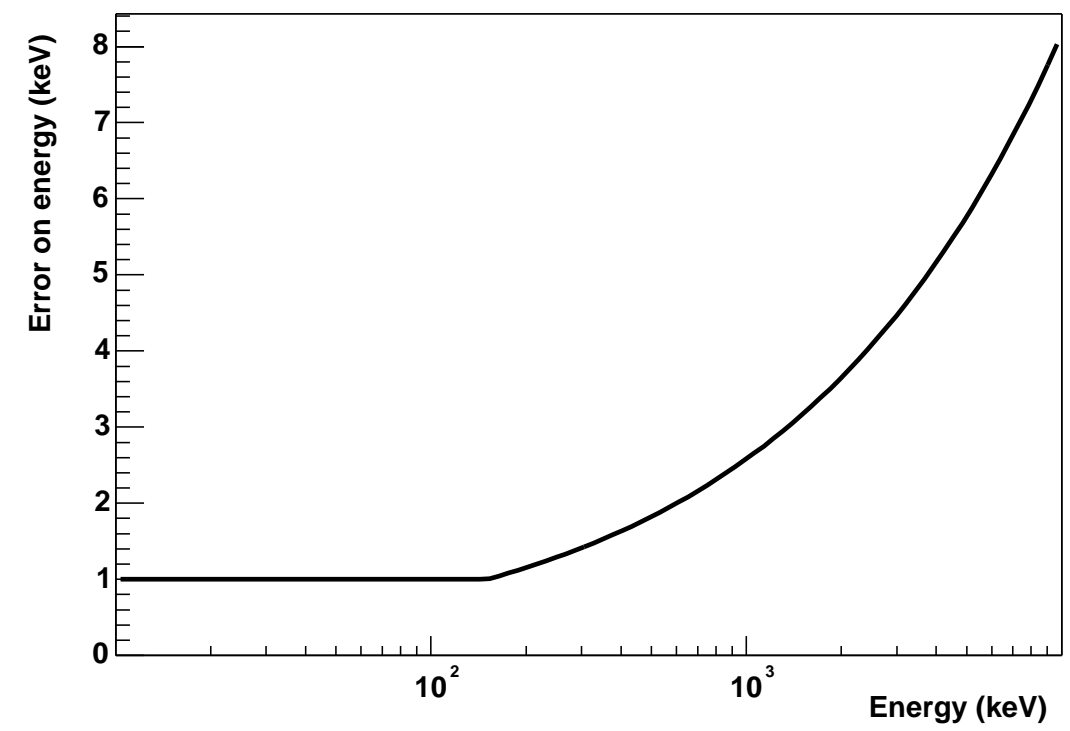

Figure 1: The simulated detector energy resolution as a function of energy deposited.

been deposited. We assume that we know the correct order of the photon interactions, and consider both photoelectric and Compton processes for the second interaction. We sum the total energy deposited in the detector to determine the incoming photon energy.

The assumed energy resolution is $2 \mathrm{keV}$ at $600 \mathrm{keV}$ with a square root energy dependence. The minimum energy resolution is $1 \mathrm{keV}$. This function is shown in Figure 1 and can be summarized as

$$
\begin{array}{r}
\delta E=1 \mathrm{keV}(E<150 \mathrm{keV}) \\
\delta E=\sqrt{E / 150 \mathrm{keV}}(E \geq 150 \mathrm{keV})
\end{array}
$$

This energy resolution is used to smear the energy deposited at each interaction site in the detector.

We then vary the position resolution from $0.1 \mathrm{~mm}$ to $10 \mathrm{~mm}$, assumed to be the same in each dimension. Given the kinematics of the generated event, the determined $d \theta$ varies considerably. For example, Figure 2 shows the $d \theta$ distribution for an input of $0.5 \mathrm{MeV}$ photons and a spatial resolution of $1 \mathrm{~mm}$. Figures 3 to 6 show the median value of $d \theta$ for initial photon energies of $0.1 \mathrm{MeV}, 0.5 \mathrm{MeV}, 1.0 \mathrm{MeV}$, and $10.0 \mathrm{MeV}$.

We observe that for spatial resolutions of the order of $1 \mathrm{~mm}$, the spatial resolution is the dominating factor in $d \theta$ for this detector configuration.

\section{Effect of events near $\theta=0$ or $\pi$}

The results reported in the previous section include all of the events, even those near $\theta=0$ or $\pi$, where the analytic expression may be overestimating the error in the Compton formula (see Appendix A.2 on page 7).

Here we show the effect of excluding these events from the analysis. Table 1 gives the fraction of events for $\theta \sim 0$ where the electron kinetic energy is within one $\sigma$ of zero $\left(E_{e}-m_{e}<\delta E_{e}\right)$, the fraction for $\theta \sim \pi$ where the scattered photon energy is with one $\sigma$ of its minimum possible energy $\left(E_{\gamma^{\prime}}<E_{\gamma^{\prime}}(\min )+\delta E_{\gamma^{\prime}}\right)$, and the median $d \theta$ with or without these events, for various incoming gammay-ray energies.

The contribution from events near $\theta=\pi$ dominates over those near $\theta=0$. Given the size of the detector, and the requirement that the events be fully contained, then the events with low energy photons are more likely to be detected. The total number of affected events is energy dependent and is approximately $5 \%$, but grows to about $20 \%$ for $100 \mathrm{keV}$ incident gamma rays. Excluding these events shifts the median $d \theta$ by less than $8 \%$ and the effect is essentially negligible for energies above about $1 \mathrm{MeV}$. 
Table 1: The effect of excluding events with $\theta \sim 0$ or $\pi$ in the calculation of $d \theta$. Shown for discrete generated energies are the fraction of events in each region and the median $d \theta$ determined including or excluding those events.

\begin{tabular}{ccccc}
\hline $\begin{array}{c}\text { Energy } \\
(\mathrm{MeV})\end{array}$ & $\begin{array}{c}\theta \sim 0 \\
(\%)\end{array}$ & $\begin{array}{c}\theta \sim \pi \\
(\%)\end{array}$ & $\begin{array}{c}d \theta \\
\text { All } \\
\text { (radians) }\end{array}$ & $\begin{array}{c}\text { Excluding } \theta \sim 0, \pi \\
\text { (radians) }\end{array}$ \\
\hline \hline 0.1 & 2.5 & 20.3 & 0.081 & 0.075 \\
0.2 & 0.5 & 11.6 & 0.037 & 0.034 \\
0.5 & 0.03 & 5.7 & 0.021 & 0.020 \\
0.8 & 0.06 & 5.3 & 0.018 & 0.017 \\
1.0 & 0 & 4.9 & 0.016 & 0.015 \\
1.33 & 0 & 3.8 & 0.016 & 0.015 \\
2.0 & 0 & 4.5 & 0.013 & 0.013 \\
3.0 & 0 & 5.0 & 0.013 & 0.013 \\
5.0 & 0 & 3.1 & 0.011 & 0.011 \\
10.0 & 0 & 3.1 & 0.011 & 0.011 \\
\hline
\end{tabular}

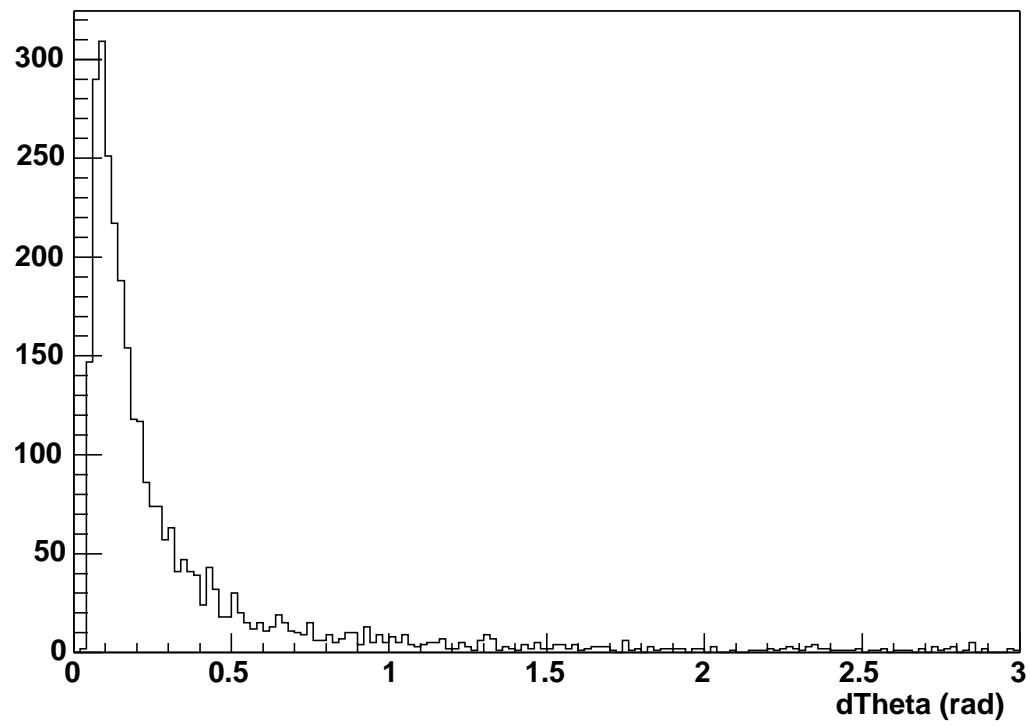

Figure 2: The $d \theta$ distribution from our simulation. The initial photon energy is $0.5 \mathrm{MeV}$ and the detector spatial resolution is simulated to be $1 \mathrm{~mm}$ in each dimension. 


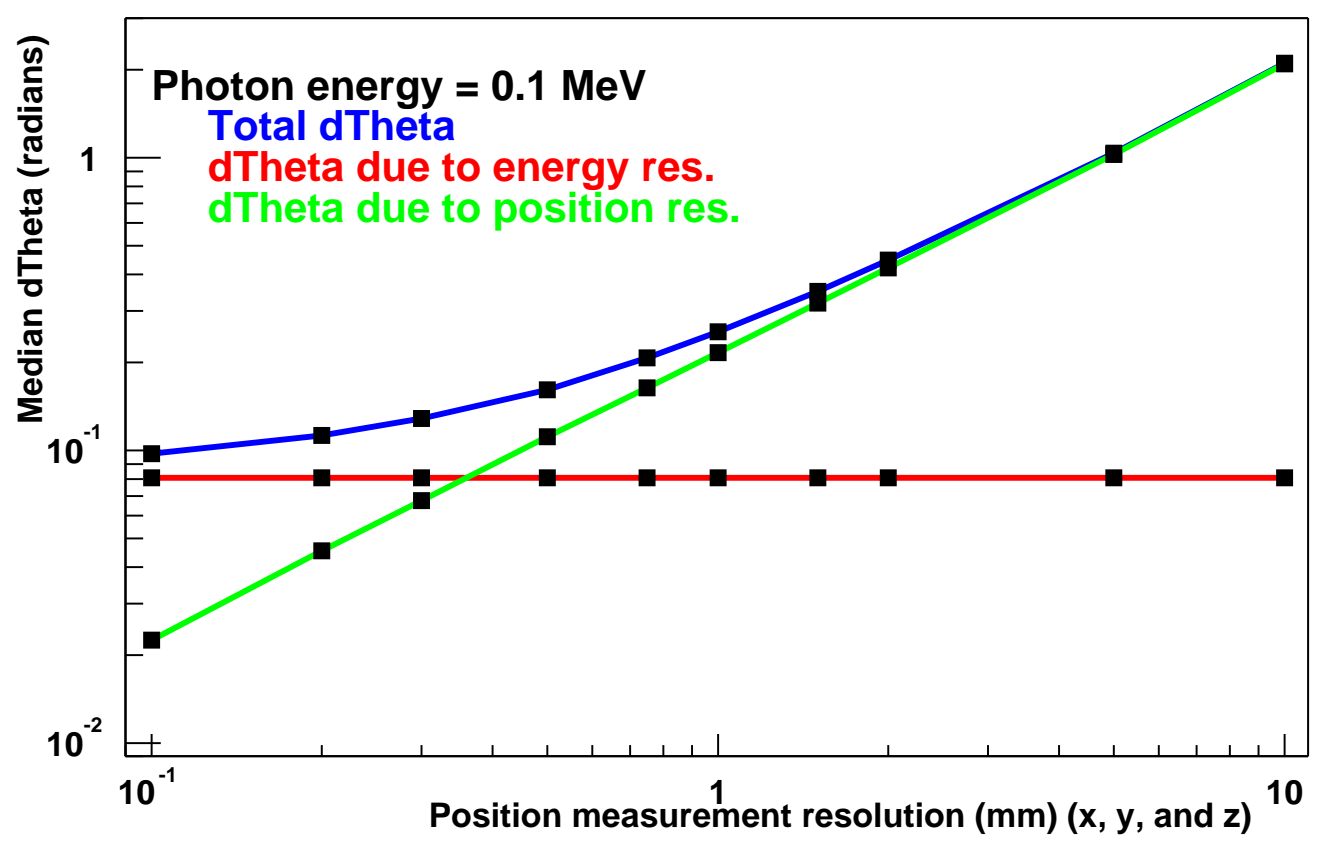

Figure 3: $d \theta$ as a function of detector spatial resolution. Plotted are the contribution to $d \theta$ due to energy resolution (red), due to spatial resolution (green), and the total $d \theta$ (blue). The input photon energy is $0.1 \mathrm{MeV}$.

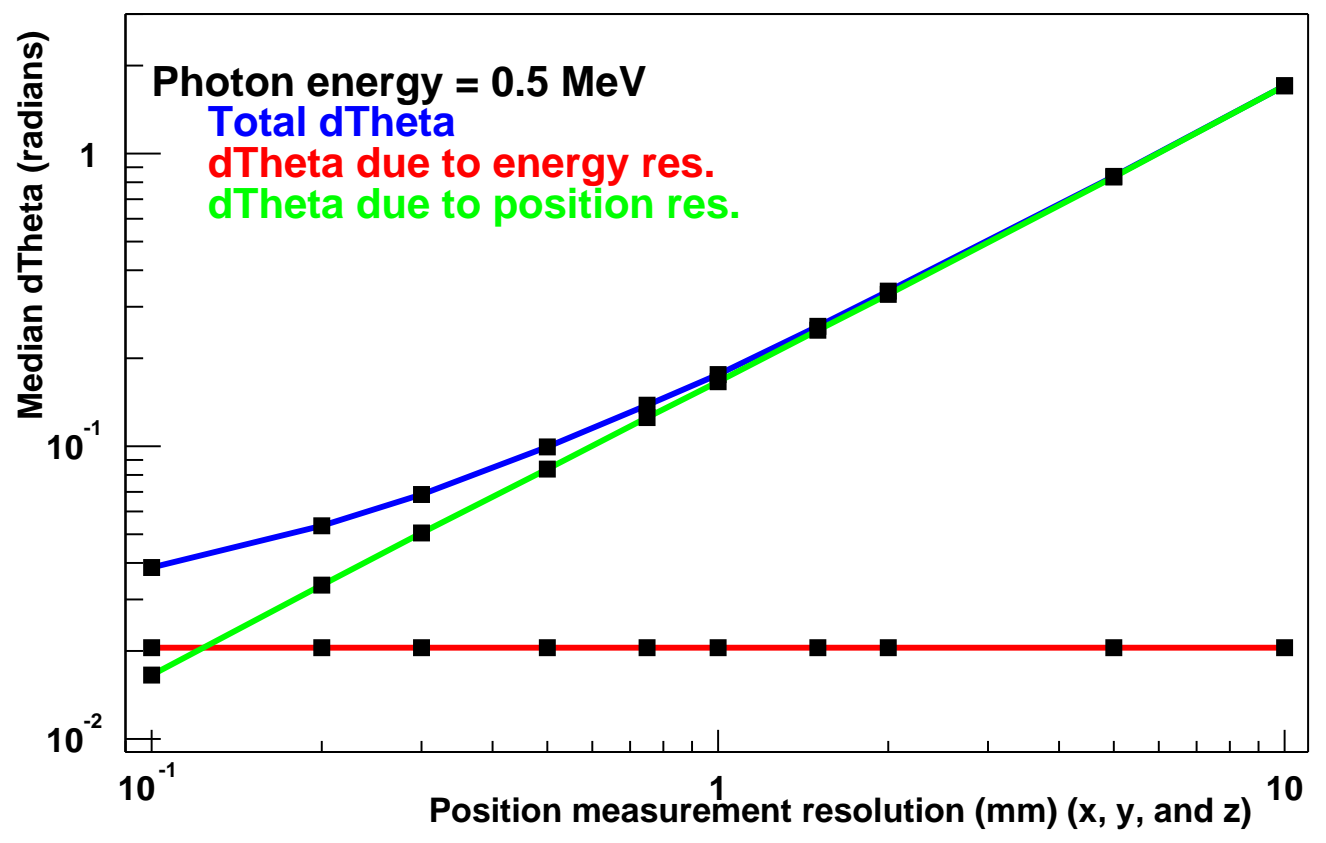

Figure 4: $d \theta$ as a function of detector spatial resolution. Plotted are the contribution to $d \theta$ due to energy resolution (red), due to spatial resolution (green), and the total $d \theta$ (blue). The input photon energy is $0.5 \mathrm{MeV}$. 


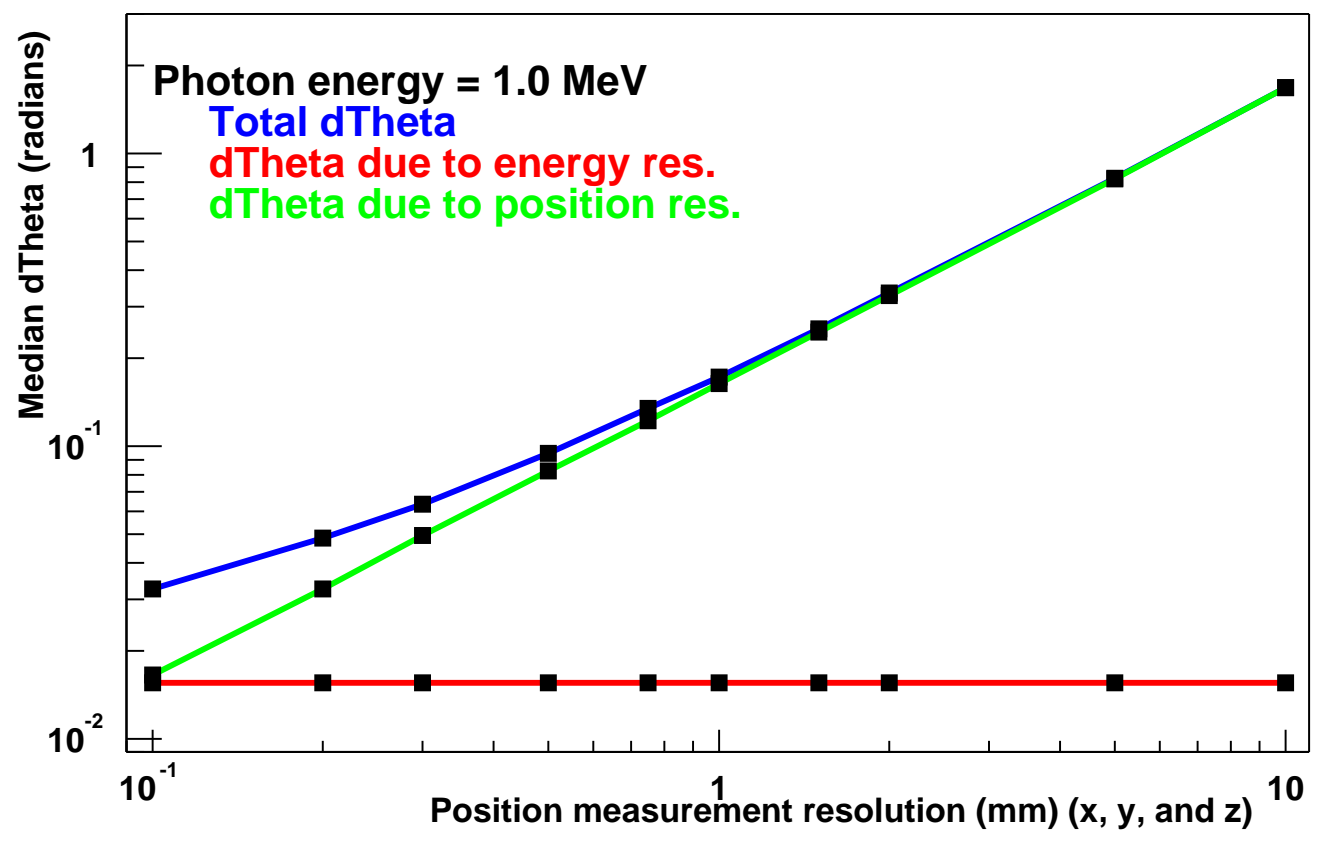

Figure 5: $d \theta$ as a function of detector spatial resolution. Plotted are the contribution to $d \theta$ due to energy resolution (red), due to spatial resolution (green), and the total $d \theta$ (blue). The input photon energy is 1 . MeV.

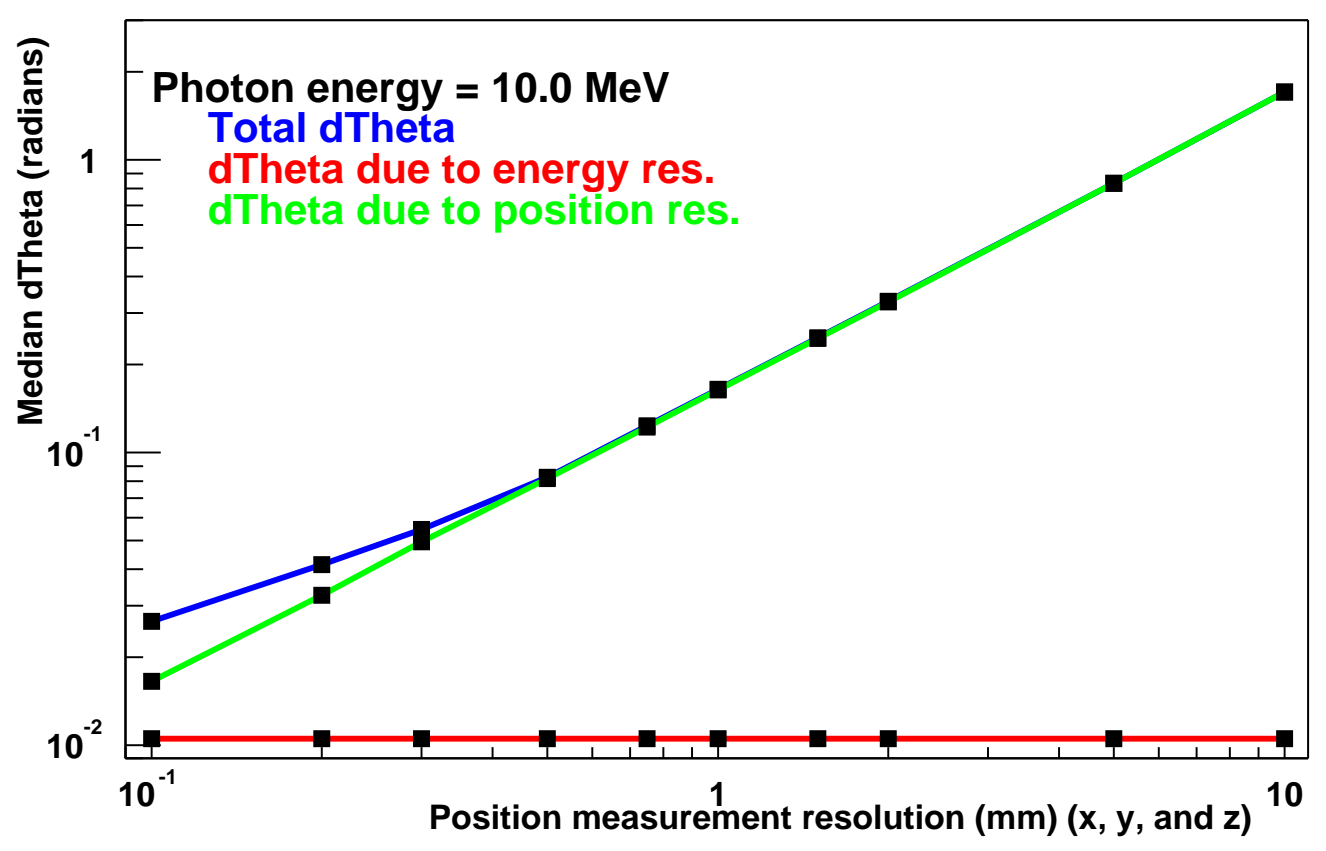

Figure 6: $d \theta$ as a function of detector spatial resolution. Plotted are the contribution to $d \theta$ due to energy resolution (red), due to spatial resolution (green), and the total $d \theta$ (blue). The input photon energy is 10. $\mathrm{MeV}$. 


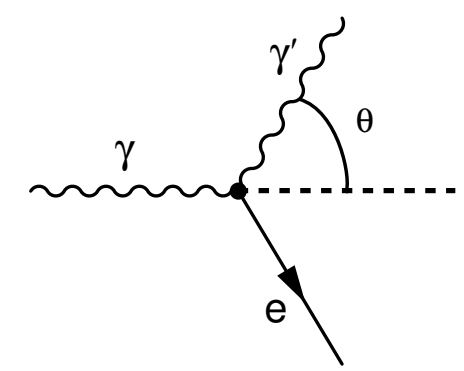

Figure 7: Compton interaction.

\section{A Appendix}

\section{A.1 Derivation of the Compton formula}

Assume that the inital electron is at rest, then from conservation of momentum, the electron recoils from the scattered photon (see Figure 7):

$$
\vec{p}_{e}=\vec{p}_{\gamma}-\vec{p}_{\gamma^{\prime}}
$$

Taking the square of the momentum and using $E_{\gamma}=p_{\gamma}$ and $E_{\gamma^{\prime}}=p_{\gamma^{\prime}}$ (since photons are massless and we are using units where $\mathrm{c}=1$ ), results in

$$
p_{e}^{2}=E_{\gamma}^{2}+E_{\gamma^{\prime}}^{2}-2 E_{\gamma} E_{\gamma^{\prime}} \cos \theta \text {. }
$$

Using the invariant mass relation $p_{e}{ }^{2}=E_{e}{ }^{2}-m_{e}{ }^{2}$ and conservation of energy $\left(E_{\gamma}+m_{e}=E_{e}+E_{\gamma^{\prime}}\right)$ to eliminate the electron variables $p_{e}$ and $E_{e}$ results in

$$
\begin{array}{r}
E_{\gamma^{\prime}}+2 m_{e} E_{\gamma}+m_{e}{ }^{2}-2 E_{\gamma} E_{\gamma^{\prime}}-2 m E_{\gamma^{\prime}}+E_{\gamma^{\prime}}{ }^{2}-m_{e}{ }^{2} \\
=E_{\gamma}{ }^{2}+E_{\gamma^{\prime}}{ }^{2}-2 E_{\gamma} E_{\gamma^{\prime}} \cos \theta
\end{array}
$$

which reduces to

$$
m_{e}\left(E_{\gamma}-E_{\gamma^{\prime}}\right)=E_{\gamma} E_{\gamma^{\prime}}(1-\cos \theta)
$$

This is often rewritten as

$$
\cos \theta=1+\frac{m_{e}}{E_{\gamma}}-\frac{m_{e}}{E_{\gamma^{\prime}}} .
$$

\section{A.1.1 Compton formula implications}

Note that the angle $\theta$ approaches zero as the electron kinetic energy $\left(E_{e}-m_{e}\right)$ approaches zero. Obviously at zero angle there is no scattering and the outgoing photon has the same energy as the incoming photon.

In contrast, the minimum outgoing photon energy can not be zero, but is instead

$$
E_{\gamma^{\prime}}(\min )=\frac{m_{e}}{2+m_{e} / E_{\gamma}}
$$

which corresponds to $\theta=\pi$, i.e., the case where the photon scatters back in the direction opposite to that of the incoming photon $\left(E_{\gamma^{\prime}}=E_{\gamma}\right)$.

Using consevation of energy $\left(E_{\gamma}=E_{\gamma^{\prime}}+E_{e}-m_{e}\right)$, one can rewrite Equation 5 in terms of the electron kinetic energy $\left(K_{e}=E_{e}-m_{e}\right)$

$$
E_{\gamma^{\prime}}(\min )=\frac{1}{2}\left(\sqrt{2 m_{e} K_{e}+K_{e}^{2}}-K_{e}\right) .
$$




\section{A.2 Analytic expression for the error in the Compton angle}

The Compton angle $\theta$ in Equation 4 depends only on the energy of the incoming $\left(E_{\gamma}=E_{\gamma^{\prime}}+E_{e}-m_{e}\right)$ and outgoing $\left(E_{\gamma^{\prime}}\right)$ gamma rays. If one considers only small Gaussian errors for the observables $\left(E_{e}\right.$ and $\left.E_{\gamma^{\prime}}\right)$, then one can derive an analytic expression for the error on $\theta$ as follows.

Taking the differential of Equation 4 yields:

$$
d(\cos \theta)=-\sin \theta d \theta=-\frac{m_{e}}{E_{\gamma}{ }^{2}} d E_{\gamma}+\frac{m_{e}}{E_{\gamma^{\prime}}{ }^{2}} d E_{\gamma^{\prime}},
$$

which can be rewritten as

$$
d \theta=\frac{m_{e}}{\sin \theta E_{\gamma}{ }^{2}}\left[d E_{\gamma}-\frac{E_{\gamma}{ }^{2}}{E_{\gamma^{\prime}}{ }^{2}} d E_{\gamma^{\prime}}\right] .
$$

Since $E_{\gamma}=E_{e}+E_{\gamma^{\prime}}-m_{e}$, we have $d E_{\gamma}=d E_{e}+d E_{\gamma^{\prime}}$, and so

$$
\begin{aligned}
d \theta & =\frac{m_{e}}{\sin \theta E_{\gamma}{ }^{2}}\left[d E_{e}+d E_{\gamma^{\prime}}-\frac{E_{\gamma}{ }^{2}}{{E_{\gamma^{\prime}}}^{2}} d E_{\gamma^{\prime}}\right] \\
& =\frac{m_{e}}{\sin \theta E_{\gamma}{ }^{2}}\left[d E_{e}+\left(1-\frac{E_{\gamma}{ }^{2}}{E_{\gamma^{\prime}}{ }^{2}}\right) d E_{\gamma^{\prime}}\right] .
\end{aligned}
$$

This equation defines the partial derivatives $\partial \theta / \partial E_{e}$ and $\partial \theta / \partial E_{\gamma^{\prime}}$. So for Gaussian errors $\delta E_{e}$ and $\delta E_{\gamma^{\prime}}$, the error estimate for $\theta$ is

$$
\delta \theta^{2}=\frac{m_{e}{ }^{2}}{\sin ^{2} \theta E_{\gamma}{ }^{4}}\left[\delta E_{e}{ }^{2}+\left(1-\frac{E_{\gamma^{2}}{ }^{2}}{E_{\gamma^{\prime}}{ }^{2}}\right)^{2} \delta E_{\gamma^{\prime}}{ }^{2}\right] .
$$

This equation is valid as long as the fractional errors $\left(\delta E_{e} / E e\right.$ and $\left.\delta E_{\gamma^{\prime}} / E_{\gamma^{\prime}}\right)$ are not too large. Furthermore, one must be careful when applying this equation in situations near $\theta=0$ or $\pi$.

\section{A.2.1 Error in Compton Angle near $\theta=0$ or $\pi$}

The case where $\theta$ is exactly zero occurs only when the electron kinetic energy $\left(K_{e}=E_{e}-m_{e}\right)$ is zero. Thus there is no detected first interaction and the formula would not be applied. Arbitrarily small values of $K_{e}$ do not cause a problem for Equation 6 until they approach the magnitude of the energy resolution itself.

For arbitrarily small values of the kinetic energy, when $K_{e} / E_{\gamma^{\prime}} \ll 1$ Equation 6 can be approximated as

$$
\delta \theta^{2} \sim \frac{m_{e} E_{\gamma^{\prime}}{ }^{2}}{2 E_{\gamma}{ }^{4} K_{e}}\left[\delta K_{e}^{2}+\frac{4 K_{e}^{2}}{E_{\gamma^{\prime}}{ }^{4}} \delta E_{\gamma^{\prime}}{ }^{2}\right] .
$$

From this one can see that $\delta \theta^{2}$ begins to blow up when the the kinetic energy becomes smaller than the energy resolution $\delta K_{e}$, but is well behaved otherwise. Note that the second term $\left(\delta E_{\gamma^{\prime}}\right)$ is suppressed for small $K_{e}$. When $K_{e}$ is smaller than about twice the energy resolution, the Gaussian approximation itself breaks down and Equation 6 will begin to overestimate the error in $\theta$. To apply this formula, one should impose a lower cutoff on the electron energy based on the energy resolution.

For the case where $\theta$ is near $\pi$, the scattered photon energy is near its minimum (but can not be zero, see Appendix A.1.1). Because of finite measurement resolution, the observed photon energy can fluctuate downward and even be lower than the minimumally allowed energy. These kinematically unallowed events will be explicitly rejected since the Compton formula itself fails. However, the case where the photon energy reaches its minimum is kinematically allowed and can also occur when events near the minimum fluctuate downard due to the detector resolution. For these events at or very near the minimum, the error formula, Equation 6, approaches infinity. To highlight the behavior near the minimum energy, we can rewrite the equation using $E_{\gamma^{\prime}}=E_{\gamma^{\prime}}(\min )(1+\delta)$, where $\delta \ll 1$.

$$
\delta \theta^{2} \sim \frac{m_{e} E_{\gamma^{\prime}}(\min )}{2 E_{\gamma}{ }^{4} \delta}\left[\delta E_{e}{ }^{2}+\left(1-\frac{E_{\gamma}{ }^{2}}{E_{\gamma^{\prime}}(\min )^{2}}(1-2 \delta)\right)^{2} \delta E_{\gamma^{\prime}}{ }^{2}\right] .
$$

Neither term $\left(\delta E_{e}, \delta E_{\gamma^{\prime}}\right)$ is suppressed, so in order to avoid this infinity, one should apply a cut to eliminate events that are less than at least one standard deviation (in terms of the energy resolution) away from the minimum photon energy. 


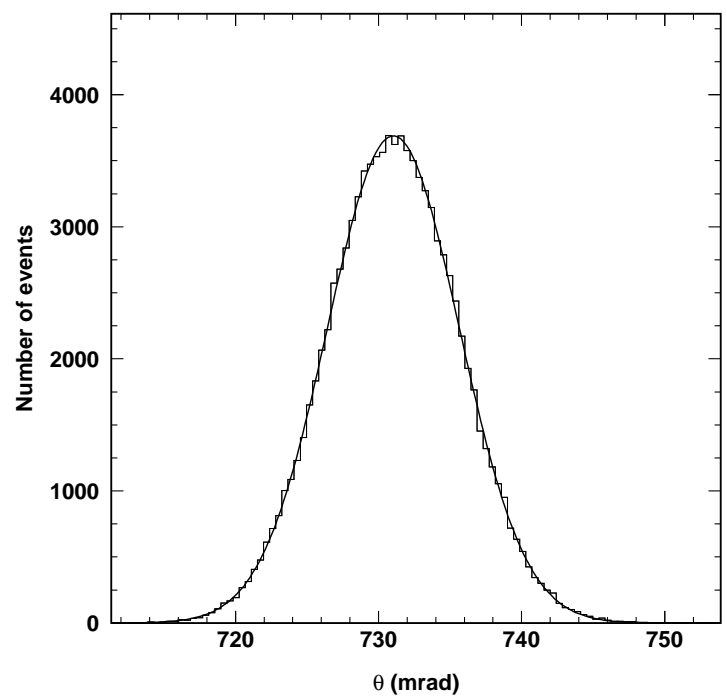

Figure 8: Reconstructed Compton angle $\theta$ from a simulation of the same physical state sampled 100,000 times with Gaussian errors for the observed energies. The fit result is superimposed on the distribution.

Table 2: Compton angle and error comparison between fit to simulation and analytic calculation. Sample of 100,000 events where the Electron kinetic energy is $100 \mathrm{keV}$ with an RMS error of $1 \mathrm{keV}$, and scattered gamma ray energy of $400 \mathrm{keV}$ with and RMS error of $2 \mathrm{keV}$.

\begin{tabular}{|l|l|l|}
\hline Variable & Fit Result & Analytic Result \\
\hline$\theta(\mathrm{mrad})$ & $731.03 \pm 0.02$ & 731.01 \\
$\delta \theta(\mathrm{mrad})$ & $4.61 \pm 0.01$ & 4.61 \\
\hline
\end{tabular}

\section{A.2.2 Numerical cross-check of Equation 6}

To check the validity of Equation 6 we performed a simple Monte Carlo analysis by generating Gaussian distributions for the input variables $E_{e}$ and $E_{\gamma^{\prime}}$ and then compared the resulting distribution of $\theta$ with the analytic expression. We generated 100,000 events where the Compton electron kinetic energy $\left(E_{e}-m_{e}\right)$ was sampled from a Gaussian distribution with a mean of $100 \mathrm{keV}$ and RMS of $1 \mathrm{keV}$, and the scattered gamma ray energy distribution had a mean of $400 \mathrm{keV}$ with an RMS of $2 \mathrm{keV}$.

Using Equation 4 we reconstruct the Compton angle $\theta$ from the simulated observables. Figure 8 shows the reconstructed $\theta$ distribution. The result of a Gaussian fit to this distribution is given in Table 2 along with the results from the analytic expressions 4 and $6 . \quad$ The agreement between the fit and the analytic expression is excellent. Be aware that the $\theta$ distribution is no longer Gaussian if the error on the energy measurement is too large, and thus Equation 6 would no longer apply. 


\section{B Angular error from position resolution}

The direction of the outgoing photon is determined from the measured positions of the two interaction points. The measurement uncertainty of these positions creates an associated uncertainty in the direction of the photon. One can derive an analytic expression that relates these uncertainties as follows.

If the unit vector $\hat{n}$ defines the direction of the incoming photon, and the two points $v_{1}=\left(x_{1}, y_{1}, z_{1}\right)$, $v_{2}=\left(x_{2}, y_{2}, z_{2}\right)$ define the direction of the outgoing photon $\vec{v}=v_{2}-v_{1}$, then the angle between the incoming and outgoing photons is

$$
\hat{n} \cdot \vec{v}=v \cos \theta=v_{\|},
$$

where $v_{\|}$is the component of $\vec{v}$ parallel to $\hat{n}$. Taking the differential of the above equation gives

$$
d v \cos \theta-\sin \theta v d \theta=d v_{\|} .
$$

Taking the differential of $v^{2}=v_{\|}^{2}+v_{\perp}^{2}$ and substituting in the above, results in

$$
v^{2} \tan \theta d \theta=v_{\perp} d v_{\perp}+v_{\|} d v_{\|}-\frac{v d v_{\|}}{\cos \theta}=v_{\perp} d v_{\perp}+\frac{\left(v_{\|} \cos \theta-v\right) d v_{\|}}{\cos \theta} .=v_{\perp} d v_{\perp}-\frac{v \sin ^{2} \theta d v_{\|}}{\cos \theta} .
$$

The error in $\theta$ is then

$$
\delta \theta^{2}=\frac{1}{v^{2} \tan ^{2} \theta}\left[v_{\perp}^{2} d v_{\perp}^{2}+\frac{v^{2} \sin ^{4} \theta}{\cos ^{2} \theta} d v_{\|}^{2}\right] .
$$

If the position resolution for each dimension is $\delta d$, then

$$
d v_{\perp}=d v_{\|}=\sqrt{2} \delta d .
$$

So $\delta \theta$ becomes

$$
\delta \theta^{2}=\frac{1}{v^{2} \tan ^{2} \theta} \frac{\left(v_{\perp}^{2} \cos ^{2} \theta+v^{2} \sin ^{4} \theta\right)}{\cos ^{2} \theta} \delta d,
$$

which reduces to

$$
\delta \theta=\frac{\sqrt{2}\left|v_{\perp}\right|}{v\left|v_{\|}\right| \tan \theta} \delta d,
$$

where $v_{\perp}\left(v_{\|}\right)$is the component of scattered photon direction, $\vec{v}$, perpendicular (parallel) to $\hat{n}$, and $\delta d$ is the position error in each measured dimension. For the special case where the incoming photon is in the $\hat{z}$ direction $(\hat{n}=(0,0,1))$, then

$$
\delta \theta=\frac{\sqrt{2} \sqrt{v_{x}^{2}+v_{y}^{2}}}{v\left|v_{z}\right| \tan \theta} \delta d .
$$

This relation holds, meaning that the resulting distribution of $\theta$ is approximately Gaussian, as long as $\delta d / p_{i}$, where $p_{i}$ is the distance between the two points in each dimension, is less than about $50 \%$. 